\title{
Somatic and sociodemographic predictors of depression outcome among depressed patients with coronary artery disease - a secondary analysis of the SPIRR-CAD study
}

Frank Vitinius ${ }^{1^{*}}$ (D), Steffen Escherich ${ }^{2 *}$, Hans-Christian Deter ${ }^{3}$, Martin Hellmich ${ }^{4}$, Jana Jünger ${ }^{5}$, Katja Petrowski ${ }^{6}$, Karl-Heinz Ladwig ${ }^{7}$, Frank Lambertus ${ }^{1}$, Matthias Michal ${ }^{8}$, Cora Weber ${ }^{3}$, Martina de Zwaan ${ }^{9}$,

Christoph Herrmann-Lingen ${ }^{10}$, Joram Ronel ${ }^{11}$ and Christian Albus ${ }^{1}$

\begin{abstract}
Background: Depressive symptoms are common in patients with coronary artery disease (CAD) and are associated with an unfavourable outcome. Establishing prognostic patient profiles prior to the beginning of mental health care may facilitate higher efficacy of targeted interventions. The aim of the current study was to identify sociodemographic and somatic predictors of depression outcome among depressed patients with CAD.

Methods: Based on the dataset of the multicentre SPIRR-CAD randomised controlled trial $(n=570$ patients with CAD and $\geq 8$ points on the Hospital Anxiety and Depression Scale (HADS)), 141 potential sociodemographic and somatic predictors of the change in the HADS-D depression score from baseline to 18-month-follow-up were derived in two different ways. We screened for univariable association with response, using either analysis of (co) variance or logistic regression, respectively, both adjusted for baseline HADS-D value and treatment group. To guard against overfitting, multivariable association was evaluated by a linear or binomial (generalised) linear model with lasso regularisation, a machine learning approach. Outcome measures were the change in continuous HADS-D depression scores, as well as three established binary criteria. The Charlson Comorbidity Index (CCl) was calculated to assess possible influences of comorbidities on our results and was also entered in our machine learning approach.

Results: Higher age $(p=0.002)$, unknown previous myocardial infarction $(p=0.013)$, and a higher heart rate variability during numeracy tests $(p=.020)$ were univariably associated with a favourable depression outcome, whereas hyperuricemia $(p \leq 0.003)$, higher triglycerides $(p=0.014)$, NYHA class III $(p \leq 0.028)$, state after resuscitation ( $p \leq 0.042$ ), intake of thyroid hormones $(p=0.007)$, antidiabetic drugs $(p=0.015)$, analgesic drugs $(p=0.027)$, beta blockers ( $p=0.035)$, uric acid drugs ( $p \leq 0.039)$, and anticholinergic drugs $(p=0.045)$ were associated with an adverse effect on the HADS-D depression score. In all analyses, no significant differences between study arms could be found and physical comorbidities also had no significant influence on our results.

(Continued on next page)
\end{abstract}

\footnotetext{
*Correspondence: frank.vitinius@uk-koeln.de; steffen.escherich@uk-koeln.de

Frank Vitinius and Steffen Escherich are joint first authors.

Joram Ronel and Christian Albus are joint last authors.

'Department of Psychosomatics and Psychotherapy, University of Cologne,

Cologne, Germany

${ }^{2}$ Department of Psychiatry and Psychotherapy, University of Cologne,

Cologne, Germany

Full list of author information is available at the end of the article
}

(c) The Author(s). 2019 Open Access This article is distributed under the terms of the Creative Commons Attribution 4.0 International License (http://creativecommons.org/licenses/by/4.0/), which permits unrestricted use, distribution, and reproduction in any medium, provided you give appropriate credit to the original author(s) and the source, provide a link to the Creative Commons license, and indicate if changes were made. The Creative Commons Public Domain Dedication waiver (http://creativecommons.org/publicdomain/zero/1.0/) applies to the data made available in this article, unless otherwise stated. 
(Continued from previous page)

Conclusion: Our findings may contribute to identification of somatic and sociodemographic predictors of depression outcome in patients with CAD. The unexpected effects of specific medication require further clarification and further research is needed to establish a causal association between depression outcome and our predictors.

Trial registration: wWW.clinicaltrials.gov NCT00705965 (registered 27th of June, 2008). wWw.isrctn.com ISRCTN76240576 (registered 27th of March, 2008).

Keywords: Depression - mental disorders - coronary heart disease - psychotherapy - type D personality

\section{Background}

Depressive symptoms are common in patients with coronary artery disease (CAD) [1-3] with an estimated prevalence of about $20 \%[1,4]$. Recent meta-analyses showed an increased risk of mortality and new cardiovascular events in CAD patients with comorbid depression [5-7]. One meta-analysis found that post-myocardial infarction depression is associated with a 2.7-fold risk of impaired cardiovascular outcome and prognosis, independent from other risk factors [8].

Besides the influential aspects of depression on CAD, the involvement of depression in the etiology of CAD has been discussed, with an adjusted effect estimate of 1.6-1.9 for CAD onset among depressed patients [6]. Due to the great importance of depression as a prognostic factor in CAD with impaired cardiovascular outcome, Lichtman et al. (2014) claim that depression should be elevated to the status of a risk factor for adverse medical outcome in patients with acute coronary syndrome [5].

The pathophysiological mechanisms underlying the association between depression and heart disease have not been proven, but there is evidence that platelet hyperreactivity and coagulation, endothelial dysfunction, inflammatory activation, autonomic and neuroendocrine dysregulation in depression might mediate the increased risk [9-12]. An explanation of the link between depression and CAD is most likely multifactorial [13]. Sociodemographic and psychosocial factors also contribute to depression and cardiovascular outcome [1].

Research has been done on the prediction of depression outcome among heterogeneous patient samples with various treatments and outcome variables. Apart from the above-mentioned sociodemographic variables, there are also somatic variables that predict treatment benefit, for example blood biomarkers such as the "brain-derived neurotrophic factor" $[14,15]$, inflammatory markers [16, 17], brain activation patterns observed by imaging studies $[18,19]$ or EEG biomarkers such as quantitative EEG measures, connectivity measures, and event-related potentials [20-22]. Many of the predictors of poor response to antidepressive treatment are at the same time cardiac risk markers, for example elevated inflammatory markers, physical inactivity or thyroid dysfunction [23, 24]. Recent reviews addressing the association of depression and CAD were limited by a lack of data on the course of depression. They mostly presume an association between specific biological or sociodemographic variables and depression at baseline, but the response to antidepressive treatment in patients with comorbid CAD has been less intensively studied.

Establishing physiological and socio-demographic variables that contribute to depression outcome among patients with CAD and thus identifying subgroups of depressed CAD patients may benefit the treatment of depression, as well as the treatment of CAD and facilitate mental health support [25]. There is substantial interest in improving clinical outcome in patients with $\mathrm{CAD}$, regarding the treatment of comorbid depression and observing its possible etiological and prognostic effects on CAD.

The main aim of the multicentre randomised controlled Stepwise Psychotherapy Intervention for Reducing Risk in Coronary Artery Disease (SPIRR-CAD) trial was to evaluate the effects of a stepwise combined short-term psychodynamic and cognitive-behavioral psychotherapy intervention on depressive symptoms in 570 patients with $\mathrm{CAD}$ and depression-scores $\geq 8$ on the Hospital Anxiety and Depression Scale (HADS-D), compared to enhanced treatment as usual (TAU) [26].

In the trial, both groups showed a decrease of depression scores but improvement in the intervention group was not superior to that found with treatment as usual. However, the intervention tended to be beneficial for depressed CAD patients with Type D personality.

Based on the dataset of the SPIRR-CAD trial [26, 27], the aim of this paper was to determine potential somatic and socio-demographic baseline predictors of depression outcome, both overall and separately for the intervention and control group and to investigate their influence on the change of depressive symptoms between baseline and 18 months. Our exploratory approach may identify further variables contributing to depression outcome, such as comorbid or other somatic conditions.

\section{Methods}

Study organisation, participants and treatment

Patients for the SPIRR-CAD trial were recruited and treated at ten different study sites across Germany (see [27] for further information). 570 patients with any 
manifestation of CAD (from stable angina to acute coronary syndrome), recent coronary angiograms and depression-scores $\geq 8$ on the Hospital Anxiety and Depression Scale (HADS-D) were randomised into two parallel arms (TOb). Exclusion criteria were severe heart failure (left ventricular ejection fraction $<20 \%$ or NYHA class IV), life-threatening conditions, severe inflammatory disease, severe depressive episodes according to SCID diagnoses, severe mental illnesses (e.g. bipolar affective disorder, psychotic disorder, dementia, persistent drug abuse) and acute suicidal tendencies. The intervention group received three sessions of individual, supportiveexpressive psychotherapy. Four to eight weeks later (T1), patients were re-evaluated and only those with persisting symptoms of depression (T2), were offered an additional 25 sessions of group psychotherapy over ten months. Patients in the control group received one 30-min tailored education session on health behaviour and psychosocial aspects of CAD. Screening for depressive symptoms was made at T0a (median $2-3$ weeks before randomisation), while more detailed diagnostic assessments were made at the baseline visit immediately before randomisation (T0b), after 4-8 weeks (T1), after 6 months (T2), 12 months (T2b), 18 months (T3) and 24 months (T4). Further information on the study organisation, sample recruitment, randomisation and treatment can be found elsewhere $[26,27]$.

\section{Outcomes}

As in the SPIRR-CAD [26] randomised controlled trial, our defined endpoint was the change in HADS-D depression scores from baseline to 18 months (T3 minus T0b), which corresponded to the end of group treatment. We supplemented our analyses, using three established binary criteria for depression. Criterion A was defined as a 50\% improvement in HADS-D depression score from TOb to T3, criterion B was defined as a HADS-D depression score lower than eight at $\mathrm{T} 3$, whereas criterion $\mathrm{C}$ represented an improvement of more than four points in the HADS-D depression score.

\section{Materials}

The dataset of the SPIRR-CAD randomised controlled trial comprised, among others, data on medical history (e.g. myocardial infarction, bypass surgery, number of events, etc.), blood tests, current drug use and socio-demographic data (e.g. education, monthly income, living with a partner, etc.). A list of all 141 screening variables can be found in an additional file [see Additional file 1, "Screening variables of the SPIRR-CAD dataset"].

\section{Charlson comorbidity index}

The Charlson Comorbidity Index (CCI) $[28,29]$ is a well validated instrument to assess the degree and burden of somatic comorbidities. It was originally designed to predict one-year mortality in patients with several comorbidities. The evaluation consists of 17 possible comorbidities that are valued from one to six points (range 0-30).

\section{Statistical analyses}

Empirical distributions of qualitative (categorical) variables were summarized by count (percentage), those of quantitative variables by either mean \pm standard deviation (SD) or median (25th to 75th percentile), depending on distributional characteristics (i.e. apparent skewness). "Univariable" associations (i.e. $p<0.05$ ) with depression outcome measures were evaluated by means of analysis of covariance and logistic regression, respectively (with assigned treatment and baseline HADS-D value as covariables). To guard against overfitting, multivariable association of all 141 variables was evaluated by a linear or binomial (generalised) linear model with lasso regularisation ( $\mathrm{R}$ package glmnet 2.0-16, a machine learning approach (see also [30]). The Charlson Comorbidity Index was also entered in the machine learning approach, representing one of the 141 variables. Beforehand, 5 datasets were completed by multiple imputation based on an iterative Markov Chain Monte Carlo method with full conditional specification and predictive mean matching. For subsequent analyses each observation was assigned a weight of $1 / 5$. The penalty parameter lambda determining feature selection was chosen by 10 -fold cross-validation (CV) to minimize mean-squared error or model deviance, respectively. Shrunken coefficients (B) or anti-logs of these $(\operatorname{Exp}(B)$, corresponding to odds ratios) are presented with $95 \%$ confidence intervals and $\log (\operatorname{lambda})$ values, the latter to describe the order of importance ("ranking") for prediction. Only the first 10 selected features (out of more than 100 variables in total) per model are presented. The selection proportion for each feature and $95 \%$ confidence intervals for $B$ and $\operatorname{Exp}(B)$ were estimated by 10,000 bootstrap replications (R package boot 1.3-20). Goodness-of-fit was characterized by mean-squared CV error and standard deviation or (predictive) odds ratio (pOR) with 95\% confidence interval, respectively. Statistical calculations were done with SPSS Statistics 25 (IBM Corp., Armonk, NY, USA) and R 3.5.0 (R Foundation for Statistical Computing, Vienna, Austria).

\section{Results}

Demographic and medical characteristics of the study cohort are shown in Table 1. Our model with lasso regularisation identified a variety of potential predictive variables of depression outcome [see Additional file 2: Table S2]. Since many coefficients (e.g. 135 for the continuous outcome measure) were non-zero, we focussed on the 10 first selected variables (i.e. with highest $\log ($ lambda) value). Since there was a big overlap with the solution of 
Table 1 Baseline characteristics by study arm

\begin{tabular}{|c|c|c|c|c|}
\hline \multirow[b]{2}{*}{ Characteristics } & \multicolumn{2}{|c|}{ Intervention group ( $n=285)$} & \multicolumn{2}{|c|}{ Control group $(n=285)$} \\
\hline & $n /$ total valid $n$ & $\%$ & $n /$ total valid $n$ & $\%$ \\
\hline \multicolumn{5}{|l|}{ Demographics } \\
\hline Age, mean \pm SD (total valid $n)$, y & $59.1 \pm 9.8(285)$ & & $59.3 \pm 9.3(285)$ & \\
\hline Female sex & $61 / 285$ & $(21.4)$ & $59 / 285$ & $(20.7)$ \\
\hline Married & $170 / 268$ & $(63.4)$ & $185 / 271$ & $(68.3)$ \\
\hline \multicolumn{5}{|l|}{ Socioeconomic status } \\
\hline Low & $117 / 285$ & $(45.0)$ & $123 / 261$ & $(47.1)$ \\
\hline Medium & $84 / 285$ & $(32.3)$ & $71 / 261$ & $(27.2)$ \\
\hline High & $59 / 285$ & (22.7) & $67 / 261$ & $(25.7)$ \\
\hline \multicolumn{5}{|l|}{ Baseline medical data } \\
\hline Hypertension & $252 / 281$ & $(89.7)$ & $245 / 280$ & $(87.5)$ \\
\hline Hyperlipidemia & $236 / 273$ & $(86.4)$ & $240 / 270$ & $(88.9)$ \\
\hline Diabetes mellitus & $69 / 275$ & $(25.1)$ & $70 / 279$ & $(25.1)$ \\
\hline $\mathrm{TSH}$, mean $\pm \mathrm{SD}$ (total valid $n), \mathrm{mU} / \mathrm{l}$ & $1.6 \pm 1.1(187)$ & & $1.5 \pm 1.2(175)$ & \\
\hline $\mathrm{BMI}$, mean $\pm \mathrm{SD}$ (total valid $n), \mathrm{kg} / \mathrm{m}^{2}$ & $28.5 \pm 5.0(280)$ & & $28.4 \pm 4.8(275)$ & \\
\hline Smokers & $90 / 282$ & (31.9) & $97 / 284$ & $(34.2)$ \\
\hline Physically active & $140 / 285$ & $(49.1)$ & $120 / 285$ & $(42.1)$ \\
\hline Previous myocardial infarction & $139 / 271$ & (51.3) & $161 / 273$ & $(59.0)$ \\
\hline Previous CABG & $53 / 283$ & $(18.7)$ & $45 / 282$ & $(16.0)$ \\
\hline Recent acute myocardial infarction & $93 / 285$ & (32.6) & $94 / 285$ & $(33.0)$ \\
\hline Recent coronary intervention ( $\mathrm{PCl}, \mathrm{CABG})$ & $204 / 285$ & (71.6) & $206 / 285$ & $(72.3)$ \\
\hline NYHA class I-II & $240 / 285$ & $(84.2)$ & $242 / 285$ & $(84.9)$ \\
\hline NYHA class III & $45 / 285$ & $(15.8)$ & $43 / 285$ & $(15.1)$ \\
\hline Charlson Comorbidity Index, median (IQR) & 2 & $(1 / 3)$ & 2 & $(1 / 3)$ \\
\hline SF-36, mean \pm SD (total valid $n$ ), & $59.0 \pm 24.9(268)$ & & $55.6 \pm 26.4(271)$ & \\
\hline \multicolumn{5}{|l|}{ Baseline medication } \\
\hline Thyroid substitution & $34 / 285$ & $(11.9)$ & $30 / 285$ & $(10.5)$ \\
\hline Continued intake (until T3) & $11 / 285$ & $(3.9)$ & $14 / 285$ & $(4.9)$ \\
\hline Uric acid lowering agents & $24 / 285$ & $(8.4)$ & $24 / 285$ & $(8.4)$ \\
\hline Antibiotics & $2 / 285$ & $(0.7)$ & $2 / 285$ & $(0.7)$ \\
\hline ACE inhibitors & $187 / 285$ & (65.6) & $193 / 285$ & $(67.7)$ \\
\hline Aspirin & $257 / 285$ & $(90.2)$ & $262 / 285$ & $(91.9)$ \\
\hline$\beta$-Blockers & $246 / 285$ & $(86.3)$ & $257 / 285$ & $(90.2)$ \\
\hline Statins & $256 / 285$ & $(89.8)$ & $265 / 285$ & $(93.0)$ \\
\hline Antidepressant medication & $33 / 285$ & (11.6) & $36 / 285$ & $(12.6)$ \\
\hline \multicolumn{5}{|c|}{ HADS-D depression subscale, mean \pm SD (total valid $n$ ) } \\
\hline Baseline & $10.4 \pm 2.5(284)$ & & $10.4 \pm 2.5(284)$ & \\
\hline After 18 months (T3) & $8.7 \pm 4.1(284)$ & & $8.9 \pm 3.9(285)$ & \\
\hline
\end{tabular}

$T S H$ Thyroid-stimulating hormone, $B M I$ body mass index, $C A B G$ coronary artery bypass graft surgery, $P C l$ percutaneous coronary intervention, NYHA New York Heart Association, IQR interquartile range, SF-36 Short Form Health Survey - physical functioning-36-item, ACE Angiotensin-converting enzyme, HADS-D Hospital Anxiety and Depression Scale - German version (depression subscale)

the "univariable" approach, any difference in coefficients may indicate the presence or amount of confounding or collinearity in the dataset.
Our continuous univariable model revealed a set of eight remaining variables significant at $p \leq .05$. Four of them could also be found with the model of lasso regularisation, 
increasing the statistical power: age $(p=.002)$, thyroid hormone substitution $(p=.007)$, higher level of triglycerides $(p=.014)$ and intake of analgesics $(p=.027)$. Higher age was associated with a favourable outcome, older patients having a significantly more reduced HADS-D score at T3, whereas thyroid hormone substitution, a higher level of triglycerides, intake of analgesics and antibiotics were associated with a significantly smaller decrease in HADS-D score. The finding concerning antibiotics could not be considered as a clinically significant influence because of the small sample size of only 4 patients who received antibiotics. The influence of higher HADS-D baseline by itself on depression outcome could be shown in the model of lasso regularisation. The study arm had no significant influence on the outcome. The CCI had no significant influence on HADS-D depression score after 18 months $(p=.238)$.

The consistent findings of both the model of criterion A (50\% improvement from baseline to T3) and of the model with lasso regularisation were unfavourably related to hyperuricemia $(p=.002)$, presence of uric acid lowering drugs $(p=.039)$, NYHA class III $(p=.028)$, and state after resuscitation $(p=.042)$. Criterion B (HADS-D score $<8$ at T3) also showed a significant, unfavourable influence of hyperuricemia $(p=.001)$, condition after resuscitation $(p<.001)$, NYHA class III $(p<.001)$ and presence of uric acid drugs $(p=.034)$. Presence of anticholinergic drugs was also associated with an adverse response after 18 months $(p=.045)$, which is in line with our results of lasso regularisation.

Criterion $\mathrm{C}$ ( $>4$ points improvement at T3) and the model with lasso regularisation indicated a negative association with hyperuricemia $(p=.003)$, presence of antidiabetic drugs $(p=.015)$ and presence of beta blockers $(p=.035)$. An unknown state after myocardial infarction $(p=.013)$ and a higher heart rate variability during numeracy tests $(p=.020)$ were associated with a better outcome. The variables "heart rate variability during dictated breathing rhythm" and "diameter of left atrium" were not interpretable because of too many missing values and an odds ratio of approximately 1 , respectively.

Several variables showed significant results in the model of the main outcome criterion (HADS-D depression score T3 minus T0b) and the additional three criteria A, B and C. These variables are highlighted with different colours in Additional file 2: Table S2.

\section{Discussion}

The purpose of this paper was to identify variables that could help to predict depression outcome and identify patients with CAD who show greater reductions of depressiveness after 18 months. Prior to this study, the extent to which somatic and socio-demographic variables predicted depression outcomes in patients with
CAD was inconclusive. Several reviews assess the association between a specific biological or socio-demographic variable and depressive symptoms at one time-point, but the analysis of their influence on depression outcome is sparse. In addition, most of the literature deals with depressed patients in general and not the specific combination of depression and comorbid CAD. There is evidence that prior depression, cardiac history and Type $\mathrm{D}$ personality are risk factors for the persistence of depressive symptoms during the first year after myocardial infarction [31].

Our model revealed significant influence of patient's age at baseline on depression change. Higher age was associated with a favourable response after 18 months. Previous literature on this topic deals with the efficacy of treatment in younger compared to older age groups but the comparison between these results is difficult. Study designs, interventions and study groups differ, as well as the number of therapy sessions or severity of depression at baseline. Few meta-analyses or reviews have taken these differences into account, in order to compare effect sizes of younger and older age groups. The authors did not find any significant difference in depression outcome between both groups [32,33], but it is important to note that they did not analyse patients with comorbid CAD, specifically. There is evidence that higher age in coronary patients is a predictor for better scores in the Mental Component Summary (MCS), a summary of all mental dimensions of the SF-36 questionnaire $[34,35]$. We have to consider that the older patients of the SPIRR-CAD dataset with comorbid CAD may differ from the younger patients, as mortality rates naturally increase with age. In addition, the older patients may have developed better coping strategies than the younger patients. Many factors that are associated with higher age and the number or severity of previous episodes of depression have to be considered $[33,36]$. In our study we evaluated depressed patients with comorbid CAD, undergoing the previously described intervention therapy. This makes it even more difficult to compare our results with others. Although our data suggest a significant effect of patient's age at baseline, the effect is small.

We detected an unexpected association between specific medications and depression outcome. Patients with thyroid hormone substitution at T0b were less likely to recover from depression. In contrast, literature shows that thyroid hormone intake may improve therapeutic response in antidepressive treatment [37-39], but it is important to note that none of these trials pay particular regards to comorbid CAD. A recent trial by Carney et al. (2016) found that high levels of the free thyroid hormone T4 predicted less improvement in depressive symptoms in patients with CAD [24]. Literature dealing 
with the association of thyroid hormone levels and depressive disorder differ in terms of analysed thyroid hormones (T3, T4, and TSH), depression rating scales, and patient characteristics (age, gender, comorbidities) with often small sample sizes. In addition, the mentioned literature evaluates treatment efficacy of depressive symptoms and is not comparable to our findings. We could only show the impact of thyroid hormones on depression outcome in general. One meta-analysis found that higher levels of the thyroid hormone thyroxine are associated with an increased risk of depression [40]. There is evidence that both hyper- and hypothyroidism are associated with changes in mood and intellectual performance $[38,40,41]$ and it is well known that especially severe hypothyroidism can imitate depressive symptoms. Our dataset included only blood levels of thyroid stimulating hormone (TSH), but we could not find a significant association between hormone levels and changes in HADS-D scores. Moreover, this variable was only available in 362 of 570 subjects (63.5\%) and further variables for assessing thyroid metabolism (T3, T4) are lacking. We do not know if our medicated patients suffer from clinical hypothyroidism or different conditions that require thyroid medication and the literature cannot be applied to our findings without restriction. A possible explanation of the negative association between depression recovery and thyroid medication intake could be a higher burden of somatic disease in patients with CAD, but it is important to note that the CCI could not show a significant influence on depressive symptoms after 18 months. There may also be possible drug interactions that cannot be detected in our study, influencing our results. Future studies should assess thyroid hormone levels (T3, T4, and TSH), as well as thyroid medication with regard to depression outcome, in order to ensure a clearer interpretation of the findings. The considerations above can also be applied to the unfavourable effect of uric acid drugs, analgesic drugs, anticholinergic drugs, antidiabetic drugs and beta blockers. There is evidence that beta blockers may be associated with an increased risk of depressive disorders, compared to other antihypertensive medication, which is consistent with our findings [42]. Altogether, the unexpected effects of specific medication require further clarification.

Our model suggests a significant association between hyperuricemia and an adverse outcome of depressive symptoms. It has been shown that elevated serum uric acid levels predict adverse outcomes after myocardial revascularisation or cardiac valve surgery [43]. Some authors maintain that the purinergic system is involved in the regulation of mood [44, 45], but current data regarding the relationship between serum uric acid levels and depression is inconsistent. Studies discovered a negative correlation between depression scores and serum uric acid levels [46-50], but one study found that hyperuricemia predispose to depression in patients with systolic heart failure, irrespective of the intake of uric acid lowering drugs [51]. In this context, it is important to note that depression and general discomfort are possible side effects of the drug "Allopurinol", a common uric acid lowering drug. Similar to the discussion of thyroid medication above, a higher burden of disease, possible drug interactions and other unexplored factors might have influenced our findings.

Our finding of the unfavourable effect of higher triglycerides, a modifiable risk factor for CAD, on depression outcome may also be explained by a higher burden of somatic disease or a reduced health-related behaviour. It is interesting to note that the BMI or other parameters of metabolism and potential comorbidities had no influence on depression outcome. Another possible indicator of a higher burden of disease may be a previous resuscitation, which could also be shown in a study of 839 patients with heart failure, predicting minor or major depression after 12 months [52].

Surprisingly, an unknown previous myocardial infarction was associated with a favourable outcome after 18 months. Patients who could not remember whether they had a myocardial infarction prior to study enrolment may show a reduced feeling of sickness in general. However, this finding remains difficult to explain.

We found an association between greater heart rate variability and a favourable depression outcome, which is in line with previous findings where heart rate variability is discussed as a marker of emotional dysregulation and predicts an increased disease risk [53-55]. One study could show that a low (nighttime) heart rate variability was a significant predictor of a poor response to treatment of major depression in patients with stable CAD [56]. Caldwell et al. (2018) suggest the use of a heart rate variability biofeedback in combination with psychotherapy, improving the outcome of depression treatment [53]. As mentioned above, we could only show possible predictors of depression outcome in general and not of treatment efficacy, highlighting a lack of comparability of the cited literature and our findings.

Several limitations must be considered when evaluating these findings. As already described in the SPIRR-CAD randomised controlled trial, severely ill patients had to be excluded and many participants had recently experienced an acute cardiac event. Therefore, our data might not be generalisable to patients with chronic stable CAD. The number of participants taking thyroid or antibiotic medication is relatively small, but to our knowledge, the analysed dataset is from the largest European treatment trial for depressed patients with $\mathrm{CAD}$ and the second largest worldwide. Besides the information on specific medication intake, further 
measures e.g. for assessing thyroid metabolism, such as T3 or T4, were not available. We also have to consider possible drug interactions that cannot be detected in our study. Moreover, though our analyses did not indicate any significant interaction of variables with the (random) treatment assignment, the identified prognostic variables for depression outcome may still be candidates for (treatment) effect modification to be further investigated in future studies. In fact, the planned analysis of the SPIRR-CAD study already showed a significant treatment-by-Type D interaction on change in depressive symptoms [26]. In the present work we wanted to detect as many potential predictors of depression outcome as possible and we therefore chose an exploratory retrospective approach. As a consequence, we have to assume some coincidental results. Strengths of this study include the large sample size and the large spectrum of analysed variables. We screened each of the 141 variables carefully and considered possible interactions between our results, as well as the influence of comorbidities.

\section{Conclusion}

The exploratory approach taken in this analysis provides information on possible predictors of depression outcome among patients with CAD. Literature concerning therapy response among this specific patient population is sparse. The evidence from the analysis should be verified in future research, analysing the unexpected influence of specific medication on depression outcome. If replicated in a future study, the consequences of the predictive information from this analysis for treatment decision-making should be considered.

\section{Additional files}

Additional file 1: Screening variables of the SPIRR-CAD dataset. (DOCX $19 \mathrm{~kb}$ )

Additional file 2: Table S2. Predictive variables of depression outcome. (XLSX $15 \mathrm{~kb})$

\footnotetext{
Abbreviations

ACE: Angiotensin-converting enzyme; ANOVA: Analysis of Variance; BMI: Body mass index; CABG: Coronary artery bypass graft surgery; CAD: Coronary artery disease; CCl: Charlson Comorbidity Index; CCS: Canadian Cardiovascular Society; EEG: Electroencephalography; HADS: Hospital Anxiety and Depression Scale; HADS-D: Hospital Anxiety and Depression Scale - German version; HRQoL: Health-Related Quality of Life; IQR: interquartile range; M: mean; MCS: Mental Component Summary; NYHA: New York Heart Association; PCI: Percutaneous coronary intervention; SCID: Structured Clinical Interview for DSM Disorders; SD: Standard deviation; SF - 36: Short Form Health Survey; SPIRR-CAD: Stepwise Psychotherapy Intervention for Reducing Risk in Coronary Artery Disease; T3: Triiodothyronine; T4: Tetraiodthyronine; TAU: Treatment as usual; TSH: Thyroid-stimulating hormone
}

\section{Funding}

The SPIRR-CAD trial has been funded by the German Research Foundation (DFG; \#HE 3115/10-1, HE 3115/10-2, AL 559/2-1, AL 559/2-2) and is supported by the German Center for Cardiovascular Research (DZHK). The Clinical Trials Center Cologne (CTC Cologne) is supported by the German Federal Ministry of Research and Education (BMBF Grant 01 KN11 06). The funding bodies did not influence the collection, analyses or interpretation of patient data.

\section{Availability of data and materials}

The datasets generated and analysed during the current study are not publicly available due to further publishing agreements but are available from the senior author on reasonable request.

\section{Authors' contributions}

FV developed the design of the secondary analysis and contributed in writing the manuscript, supported SE conducting the literature research and was actively involved in collecting, analysing and interpreting the patient data. SE was the major contributor in writing the manuscript and supported FV in developing the design of the secondary analysis. In addition, SE conducted the literature research and was actively involved in collecting, analysing and interpreting the patient data. The authors wish it to be known that, in their opinion, the first two authors should be regarded as joint First Authors. CHL and CA were the coordinating investigators of the main SPIRRCAD trial, contributed to the manuscript and were actively involved in collecting, analyzing and interpreting the patient data. $\mathrm{MH}$ was the major contributor in the statistical analysis. He was actively involved in collecting, analyzing and interpreting the patient data. JR contributed to the design of the study and the manuscript. He was actively involved in collecting, analysing and interpreting the patient data. HCD, KHL, MM, MZ, JJ, KP, FL and CW contributed to the manuscript and were actively involved in collecting, analysing and interpreting the patient data. All authors read and approved the final manuscript.

\section{Ethics approval and consent to participate}

Research was approved by the Ethics Commission of the University Medical Faculty in Göttingen (Cologne 08-182, Göttingen 05/10/07). Written informed consent to participate in the study was obtained from all participants.

\section{Consent for publication}

Not applicable.

\section{Competing interests}

The authors declare that they have no competing interests. C. HerrmannLingen is receiving royalties for the German version of the HADS.

\section{Publisher's Note}

Springer Nature remains neutral with regard to jurisdictional claims in published maps and institutional affiliations.

\section{Author details}

${ }^{1}$ Department of Psychosomatics and Psychotherapy, University of Cologne, Cologne, Germany. ${ }^{2}$ Department of Psychiatry and Psychotherapy, University of Cologne, Cologne, Germany. ${ }^{3}$ Department of Psychosomatics and Psychotherapy, Charité Universitaetsmedizin Berlin, Campus Benjamin Franklin, Berlin, Germany. ${ }^{4}$ Institute of Medical Statistics and Computational Biology (IMSB), University of Cologne, Cologne, Germany. ${ }^{5}$ German National Institute for state examinations in Medicine, Pharmacy and Psychotherapy, Mainz, Germany. 'Department of Psychotherapy and Psychosomatic Medicine, Technical University Dresden, Dresden, Germany. ${ }^{7}$ German Research Center of Environmental Health, Helmholtz Zentrum Muenchen, Institute of Epidemiology, Oberschleißheim, Germany. ${ }^{8}$ Department of Psychosomatic Medicine and Psychotherapy, University Hospital of Mainz, Mainz, Germany. ${ }^{9}$ Department of Psychosomatic Medicine and Psychotherapy, Hannover Medical School, Hannover, Germany. ${ }^{10}$ Department of Psychosomatic Medicine and Psychotherapy, University of Goettingen Medical Center and German Center for Cardiovascular Research (DZHK), Partner Site Goettingen, Goettingen, Germany. ${ }^{11}$ Department of Psychosomatic Medicine and Psychotherapy, University Hospital Rechts der Isar, Technische Universitaet München, Munich, Germany. 
Received: 5 December 2017 Accepted: 15 January 2019 Published online: 04 February 2019

\section{References}

1. Ladwig KH, Lederbogen F, Albus C, Angermann C, Borggrefe M, Fischer D, et al. Position paper on the importance of psychosocial factors in cardiology: Update 2013. German medical science : GMS e-journal. 2014;12: Doc09.

2. Shanmugasegaram S, Russell KL, Kovacs AH, Stewart DE, Grace SL. Gender and sex differences in prevalence of major depression in coronary artery disease patients: a meta-analysis. Maturitas. 2012;73:305-11.

3. Ormel J, Von Korff M, Burger H, Scott K, Demyttenaere K, Huang YQ, et al. Mental disorders among persons with heart disease - results from world mental health surveys. Gen Hosp Psychiatry. 2007;29:325-34.

4. Thombs BD, Bass EB, Ford DE, Stewart KJ, Tsilidis KK, Patel U, et al. Prevalence of depression in survivors of acute myocardial infarction. J Gen Intern Med. 2006;21:30-8.

5. Lichtman JH, Froelicher ES, Blumenthal JA, Carney RM, Doering LV, FrasureSmith $\mathrm{N}$, et al. Depression as a risk factor for poor prognosis among patients with acute coronary syndrome: systematic review and recommendations: a scientific statement from the American Heart Association. Circulation. 2014;129:1350-69.

6. Ladwig KH, Emeny RT, Häfner S, Lacruz ME. Depression. Bundesgesundheitsbl Gesundheitsforsch Gesundheitsschutz. 2011;54:59-65.

7. Meijer A, Conradi HJ, Bos EH, Anselmino M, Carney RM, Denollet J, et al. Adjusted prognostic association of depression following myocardial infarction with mortality and cardiovascular events: individual patient data meta-analysis. Br J Psychiatry. 2013;203:90-102.

8. Meijer A, Conradi HJ, Bos EH, Thombs BD, van Melle JP, de Jonge P. Prognostic association of depression following myocardial infarction with mortality and cardiovascular events: a meta-analysis of 25 years of research. Gen Hosp Psychiatry. 2011;33:203-16.

9. Granville Smith I, Parker G, Rourke P, Cvejic E, Vollmer-Conna U. Acute coronary syndrome and depression: a review of shared pathophysiological pathways. Aust N Z J Psychiatry. 2015.

10. Davidson KW. Depression and coronary heart disease. ISRN cardiology. 2012; 2012:743813.

11. Penninx BW, Milaneschi Y, Lamers F, Vogelzangs N. Understanding the somatic consequences of depression: biological mechanisms and the role of depression symptom profile. BMC Med. 2013;11:129.

12. Dickens $C$. Depression in people with coronary heart disease: prognostic significance and mechanisms. Curr Cardiol Rep. 2015;17:83.

13. Frasure-Smith $N$, Lesperance $F$. Depression and cardiac risk: present status and future directions. Postgrad Med J. 2010;86:193-6.

14. Molendijk ML, Spinhoven P, Polak M, Bus BA, Penninx BW, Elzinga BM. Serum BDNF concentrations as peripheral manifestations of depression: evidence from a systematic review and meta-analyses on 179 associations ( $N=9484)$. Mol Psychiatry. 2014;19:791-800.

15. Molendijk ML, Bus BA, Spinhoven P, Penninx BW, Kenis G, Prickaerts J, et al. Serum levels of brain-derived neurotrophic factor in major depressive disorder: state-trait issues, clinical features and pharmacological treatment. Mol Psychiatry. 2011;16:1088-95.

16. Howren MB, Lamkin DM, Suls J. Associations of depression with C-reactive protein, IL-1, and IL-6: a meta-analysis. Psychosom Med. 2009;71:171-86.

17. Valkanova V, Ebmeier KP, Allan CL. CRP, IL-6 and depression: a systematic review and meta-analysis of longitudinal studies. J Affect Disord. 2013;150: 736-44.

18. MacQueen GM. Magnetic resonance imaging and prediction of outcome in patients with major depressive disorder. J Psychiatry Neurosci. 2009;34:343-9.

19. Fu CH, Steiner H, Costafreda SG. Predictive neural biomarkers of clinical response in depression: a meta-analysis of functional and structural neuroimaging studies of pharmacological and psychological therapies. Neurobiol Dis. 2013;52:75-83.

20. Olbrich S. Arns M. EEG biomarkers in major depressive disorder: discriminative power and prediction of treatment response. Int Rev Psychiatry. 2013;25:604-18.

21. Korb AS, Hunter AM, Cook IA, Leuchter AF. Rostral anterior cingulate cortex theta current density and response to antidepressants and placebo in major depression. Clin Neurophysiol. 2009;120:1313-9.
22. Leuchter AF, Cook IA, Marangell LB, Gilmer WS, Burgoyne KS, Howland RH, et al. Comparative effectiveness of biomarkers and clinical indicators for predicting outcomes of SSRI treatment in major depressive disorder: results of the BRITE-MD study. Psychiatry Res. 2009;169:124-31.

23. Carney RM, Freedland KE, Miller GE, Jaffe AS. Depression as a risk factor for cardiac mortality and morbidity: a review of potential mechanisms. J Psychosom Res. 2002;53:897-902.

24. Carney RM, Freedland KE, Steinmeyer B, Rubin EH, Mann DL, Rich MW. Cardiac risk markers and response to depression treatment in patients with coronary heart disease. Psychosom Med. 2016;78:49-59.

25. Whalley B, Thompson DR, Taylor RS. Psychological interventions for coronary heart disease: cochrane systematic review and meta-analysis. Int J Behav Med. 2014;21:109-21.

26. Herrmann-Lingen C, Beutel ME, Bosbach A, Deter HC, Fritzsche K, Hellmich $M$, et al. A stepwise psychotherapy intervention for reducing risk in coronary artery disease (SPIRR-CAD): results of an observer-blinded, multicenter, randomized trial in depressed patients with coronary artery disease. Psychosom Med. 2016;78:704-15.

27. Albus C, Beutel ME, Deter HC, Fritzsche K, Hellmich M, Jordan J, et al. A stepwise psychotherapy intervention for reducing risk in coronary artery disease (SPIRR-CAD) - rationale and design of a multicenter, randomized trial in depressed patients with CAD. J Psychosom Res. 2011;71:215-22.

28. Charlson ME, Pompei $P$, Ales KL, Mackenzie CR. A new method of classifying prognostic comorbidity in longitudinal studies: development and validation. J Chronic Dis. 1987;40:373-83.

29. Sundararajan V, Henderson T, Perry C, Muggivan A, Quan H, Ghali WA. New ICD-10 version of the Charlson comorbidity index predicted in-hospital mortality. J Clin Epidemiol. 2004;57:1288-94.

30. Kessler RC, van Loo HM, Wardenaar KJ, Bossarte RM, Brenner LA, Cai T, et al. Testing a machine-learning algorithm to predict the persistence and severity of major depressive disorder from baseline self-reports. Mol Psychiatry. 2016;21:1366-71.

31. Martens EJ, Smith OR, Winter J, Denollet J, Pedersen SS. Cardiac history, prior depression and personality predict course of depressive symptoms after myocardial infarction. Psychol Med. 2008;38:257-64.

32. Cuijpers P, van Straten A, Smit F. Andersson G. Is psychotherapy for depression equally effective in younger and older adults? A meta-regression analysis. Int Psychogeriatr. 2009;21:16-24.

33. Mitchell AJ, Subramaniam H. Prognosis of depression in old age compared to middle age: a systematic review of comparative studies. Am J Psychiatry. 2005;162:1588-601.

34. Bengtsson I, Hagman M, Wedel $\mathrm{H}$. Age and angina as predictors of quality of life after myocardial infarction: a prospective comparative study. Scand Cardiovasc J. 2001;35:252-8.

35. Soto M, Failde I, Márquez S, Benítez E, Ramos I, Barba A, et al. Physical and mental component summaries score of the SF-36 in coronary patients. Qual Life Res. 2005;14:759-68.

36. Brown N, Melville M, Gray D, Young T, Munro J, Skene AM, et al. Quality of life four years after acute myocardial infarction: short form 36 scores compared with a normal population. Heart. 1999;81:352-8.

37. Posternak M, Novak S, Stern R, Hennessey J, Joffe R, Prange A Jr, et al. A pilot effectiveness study: placebo-controlled trial of adjunctive Ltriiodothyronine (T3) used to accelerate and potentiate the antidepressant response. Int J Neuropsychopharmacol. 2008;11:15-25.

38. Bauer $\mathrm{M}$, London ED, Silverman DH, Rasgon N, Kirchheiner J, Whybrow PC. Thyroid, brain and mood modulation in affective disorder: insights from molecular research and functional brain imaging. Pharmacopsychiatry. 2003; 36(Suppl 3):S215-21.

39. Altshuler LL, Bauer M, Frye MA, Gitlin MJ, Mintz J, Szuba MP, et al. Does thyroid supplementation accelerate tricyclic antidepressant response? A review and meta-analysis of the literature. Am J Psychiatry. 2001;158:1617-22.

40. Williams MD, Harris R, Dayan CM, Evans J, Gallacher J, Ben-Shlomo Y. Thyroid function and the natural history of depression: findings from the Caerphilly prospective study (CaPS) and a meta-analysis. Clin Endocrinol. 2009;70:484-92.

41. Stipcevic T, Pivac N, Kozaric-Kovacic D, Muck-Seler D. Thyroid activity in patients with major depression. Coll Antropol. 2008;32:973-6.

42. Boal AH, Smith DJ, McCallum L, Muir S, Touyz RM, Dominiczak AF, et al. Monotherapy with major antihypertensive drug classes and risk of hospital admissions for mood disorders. Hypertension (Dallas, Tex : 1979). 2016;68: 1132-8. 
43. Lazzeroni D, Bini M, Camaiora U, Castiglioni P, Moderato L, Bosi D, et al. Serum uric acid level predicts adverse outcomes after myocardial revascularization or cardiac valve surgery. Eur J Prev Cardiol. 2018;25:119-26.

44. Ortiz R, Ulrich H, Zarate CA Jr, Machado-Vieira R. Purinergic system dysfunction in mood disorders: a key target for developing improved therapeutics. Prog Neuro-Psychopharmacol Biol Psychiatry. 2015;57:117-31.

45. Jinnah HA. Lesch-Nyhan disease: from mechanism to model and back again. Dis Model Mech. 2009;2:116-21.

46. Kesebir S, Tatlidil Yaylaci E, Suner O, Gultekin BK. Uric acid levels may be a biological marker for the differentiation of unipolar and bipolar disorder: the role of affective temperament. J Affect Disord. 2014;165:131-4.

47. Chaudhari K, Khanzode S, Khanzode S, Dakhale G, Saoji A, Sarode S. Clinical correlation of alteration of endogenous antioxidant-uric acid level in major depressive disorder. Indian J Clin Biochem: IJCB. 2010;25:77-81.

48. Bove M, Carnevali L, Cicero AF, Grandi E, Gaddoni M, Noera G, et al. Psychosocial factors and metabolic parameters: is there any association in elderly people? The Massa Lombarda project. Aging Ment Health. 2010;14: 801-6.

49. Liu T, Zhong S, Liao X, Chen J, He T, Lai S, et al. A meta-analysis of oxidative stress markers in depression. PLoS One. 2015;10:e0138904.

50. Wen S, Cheng M, Wang H, Yue J, Wang H, Li G, et al. Serum uric acid levels and the clinical characteristics of depression. Clin Biochem. 2012:45:49-53.

51. Angermann CE, Gelbrich G, Stork S, Schowalter M, Deckert J, Ertl G, et al. Somatic correlates of comorbid major depression in patients with systolic heart failure. Int J Cardiol. 2011;147:66-73.

52. Lossnitzer N, Herzog W, Stork S, Wild B, Muller-Tasch T, Lehmkuhl E, et al. Incidence rates and predictors of major and minor depression in patients with heart failure. Int J Cardiol. 2013;167:502-7.

53. Caldwell YT, Steffen PR. Adding HRV biofeedback to psychotherapy increases heart rate variability and improves the treatment of major depressive disorder. Int J Psychophysiol. 2018;131:96-101.

54. Kemp AH, Quintana DS, Gray MA, Felmingham KL, Brown K, Gatt JM. Impact of depression and antidepressant treatment on heart rate variability: a review and meta-analysis. Biol Psychiatry. 2010;67:1067-74.

55. Sgoifo A, Carnevali L, Alfonso Mde L, Amore M. Autonomic dysfunction and heart rate variability in depression. Stress. 2015;18:343-52.

56. Carney RM, Freedland KE, Steinmeyer BC, Rubin EH, Stein PK, Rich MW. Nighttime heart rate predicts response to depression treatment in patients with coronary heart disease. J Affect Disord. 2016;200:165-71.

Ready to submit your research? Choose BMC and benefit from:

- fast, convenient online submission

- thorough peer review by experienced researchers in your field

- rapid publication on acceptance

- support for research data, including large and complex data types

- gold Open Access which fosters wider collaboration and increased citations

- maximum visibility for your research: over $100 \mathrm{M}$ website views per year

At BMC, research is always in progress.

Learn more biomedcentral.com/submissions 